\title{
BRD4 inhibition alleviates mechanical stress-induced TMJ OA-like pathological changes and attenuates TREM1-mediated inflammatory response
}

\author{
Ziwei Huang ${ }^{1 \dagger}$, Ren Yang ${ }^{1 \dagger}$, Lu Zhang ${ }^{1}$, Mengjiao Zhu ${ }^{2}$, Caixia Zhang ${ }^{1}$, Juan Wen ${ }^{1}$ and Huang $\mathrm{Li}^{3^{*}}$
}

\begin{abstract}
The aim of this paper was to investigate the protective effects of bromodomain containing 4 (BRD4) inhibition on the temporomandibular joint osteoarthritis (TMJ OA) induced by compressive mechanical stress and to explore the underlying mechanism. In vivo, a rat model of TMJ compressive loading device was used and BRD4 inhibitor was injected into the TMJ region. HE staining and micro-CT analysis were used for histological and radiographic assessment. Immunohistochemistry and qPCR were performed to detect inflammatory cytokines expressions. High-throughput ChIP-sequencing screening was performed to compare the BRD4 and H3K27ac binding patterns between condylar cartilage from control and mechanical force groups. In vitro, the mandibular condylar chondrocytes were treated with IL-1 $\beta$. Small Interference RNA (siRNA) infection was used to silencing BRD4 or TREM1. qPCR was performed to detect inflammatory cytokines expressions. Our study showed that BRD4 inhibition can alleviate the thinning of condylar cartilage and subchondral bone resorption, as well as decrease the inflammatory factors expression both in vivo and in vitro. ChIP-seq analysis showed that BRD4 was more enriched in the promoter region of genes related to the stress and inflammatory pathways under mechanical stress in vivo. Trem1, a pro-inflammatory gene, was screened out from the overlapped BRD4 and H3K27ac increased binding sites, and Trem 1 mRNA was found to be regulated by BRD4 inhibition both in vivo and in vitro. TREM1 inhibition reduced the expression of inflammatory factors induced by IL-1 $\beta$ in vitro. In summary, we concluded that BRD4 inhibition can protect TMJ OA-like pathological changes induced by mechanical stress and attenuate TREM1-mediated inflammatory response.
\end{abstract}

Keywords: TMJ, Osteoarthritis, BRD4, TREM1, Inflammation

\section{Introduction}

Temporomandibular joint osteoarthritis (TMJ OA) is a traditionally defined degenerative joint disorder. It has been proved that excessive mechanical stress is an important etiological factor of TMJ OA [1-4]. We have successfully constructed the TMJ OA rat model induced by

\footnotetext{
*Correspondence: lihuang76@nju.edu.cn

†Ziwei Huang and Ren Yang contributed equally to this work

${ }^{3}$ Department of Orthodontics, Nanjing Stomatological Hospital, Medical School of Nanjing University, 30 Central Road, Nanjing 210008, China

Full list of author information is available at the end of the article
}

compressive mechanical stress [5-10]. Previous studies reported that the thinning of the cartilage, subchondral bone resorption, and extracellular matrix (ECM) damage are the main pathological changes induced by excessive mechanical stress [7-9]. Recently, researchers began to focus on the role of inflammation in TMJ OA [11-13]. From the traditionally defined degenerative disease to the present aseptic inflammatory disease, the mechanism of these pathological changes is still unclear.

More and more studies found that many human diseases are associated with epigenetic changes [13-15]. BRD4 is a double bromodomain protein of the BET 
family which binds the acetylated histones and regulates transcription as an epigenetic reader [16]. BRD4 is considered as a potential therapeutic target in many inflammatory diseases [17-22]. It was reported that BRD4 has dual effects on the HMGB1 and NF- $\mathrm{KB}$ signaling pathways [18] and BRD4 can modulate macrophage inflammatory responses [17]. BRD4 can also regulate osteoclast differentiation and was involved in the osteoporosis [14, $23,24]$. In recent years, several BRD4 inhibitors such as JQ1 were developed and proved to inhibit inflammation through blocking the interaction between BRD4 and acetylated histones $[17-19,25,26]$. Thereby, whether BRD4-mediated inflammatory response also plays a key role in TMJ OA sparked our interest.

Based on previous studies, we hypothesized that BRD4 inhibition may alleviate the TMJ OA-like pathological changes induced by excessive mechanical stress. To prove this hypothesis and explore the underlying mechanism, we designed in vitro IL-1 $\beta$ treatment model and in vivo rat model of TMJ compressive loading device with the use of BRD4 inhibitor JQ1 and siRNA interference. We found that BRD4 inhibition can alleviate the thinning of condylar cartilage expression and decrease the inflammatory factors. ChIP-seq analysis also showed that BRD4 was more enriched in the promoter region of genes related to the stress and inflammatory pathways under mechanical stress. Trem1 was screened out from the overlapped binding sites of BRD4 and H3K27ac ChIPseq. We demonstrated that BRD4 inhibition can reduce the TREM1-mediated inflammatory response after overloading mechanical stress or IL-1 $\beta$ treatment.

\section{Result \\ BRD4 inhibition can relieve the condylar cartilage thinning and subchondral bone resorption induced by excessive mechanical stress in vivo}

To explore whether BRD4 inhibition has a protective effect on cartilage under excessive mechanical stress, we set up 4 experimental groups including control group (Control), JQ1 group, mechanical force group (MF) and mechanical force plus JQ1 injection group (MF+JQ1) (Fig. 1a). JQ1 can block the interaction between BRD4 and acetylated histones and was used as the BRD4 inhibitor [27]. HE results showed that the control group had thicker cartilage and clear structure. The fibrous proliferative layer (FP), the transition layer $(\mathrm{T})$, and the hypertrophic layer $(\mathrm{H})$ were clearly layered, and the trabecular bone was dense in subchondral bone. The thickness and morphology of the cartilage in the JQ1 group were basically the same as those in the control group. After 14 days of overloading, the cartilage thickness reduced by $60 \%$ compared with the control group and apparent subchondral bone destruction was found with marked inflammatory cells infiltration near the dilated blood vessels (Fig. 1b). BRD4 inhibitor JQ1 can relieve the cartilage thinning induced by excessive mechanical stress. Consistent with the thickness of the cartilage, the number of chondrocytes also decreased in MF group and increased in MF+JQ1 groups compared to MF group (Fig. 1c). These results suggested that BRD4 inhibitor JQ1 can effectively reduce the condylar cartilage thinning and chondrocytes loss caused by excessive mechanical stress.

Micro-CT examination can reflect the changes of condylar subchondral bone morphology in a more stereoscopic and intuitive way from three dimensions (Fig. 1d). The results showed that the ratio of bone volume to tissue volume (BV/TV), trabecular number (Tb.N.), and trabecular thickness (Tb.Th.) decreased and trabecular separation (Tb.Sp.) increased in the MF group. In the MF + JQ1 group, the BV/TV increased by $27 \%(P<0.01)$ and the Tb.Th increased by $9 \%$ compared with the MF group (Fig. 1e). It is indicated that JQ1 can inhibit the condylar subchondral bone resorption and is beneficial to maintain the normal morphological structure of subchondral bone under excessive mechanical stress.

\section{BRD4 inhibition can alleviate the expression of inflammatory factors induced by excessive mechanical stress in vivo and IL-1 $\beta$ in vitro}

To confirm whether BRD4 inhibition can inhibit the cartilage inflammation induced by mechanical stress, we detected the expression of inflammatory factors by qRTPCR and immunohistochemistry in vivo. The mRNA level of Tnf- $\alpha, I l-1 \beta$ and $I l-6$ showed no significant difference in the control group and JQ1 group. After 14 days of overloading, the above genes expression increased significantly. JQ1 can reduce the Tnf- $\alpha, I l-1 \beta$, and Il-6 mRNA expression (Fig. 2a). Next, we performed TNF- $\alpha$ and IL-1 $\beta$ immunohistochemical staining and scoring. The results corresponded to those of qRT-PCR (Fig. 2b). These results indicated that BRD4 inhibitor JQ1 can inhibit the expression of inflammatory factors in rat condylar cartilage induced by mechanical stress. Previous studies have reported that IL- $1 \beta$ triggers proinflammatory factor production in chondrocytes $[11,28$, 29 ] and IL- $1 \beta$ plays a vital role in TMJ OA development $[11,30]$. Therefore, we used IL-1 $\beta$ as the stimuli to study the anti-inflammation effect of BRD4 inhibition in vitro. The efficiency of siBRD4 was detected as shown in Additional file 1: Fig. S2. We found that both JQ1 and siBRD4 can inhibit the increase of $B r d 4, I l-6$, Tnf- $\alpha$ mRNA and endogenous $I l-1 \beta$ mRNA induced by IL-1 $\beta$ in vitro (Fig. 2c). 


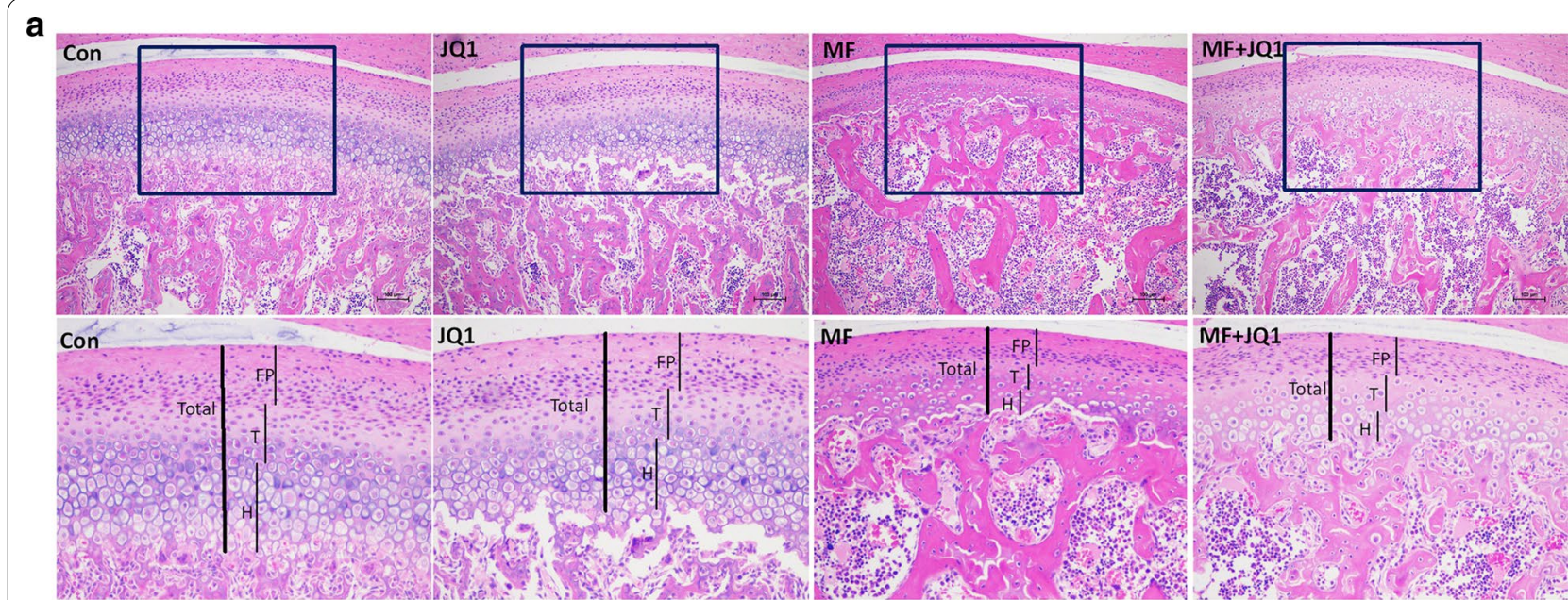

b

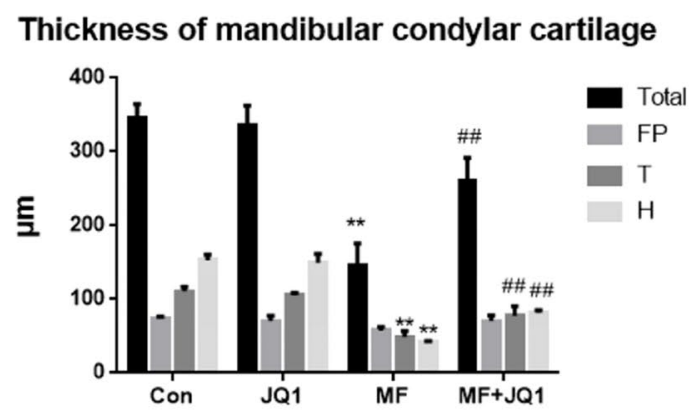

C

\section{Number of mandibular condylar chondrocytes}

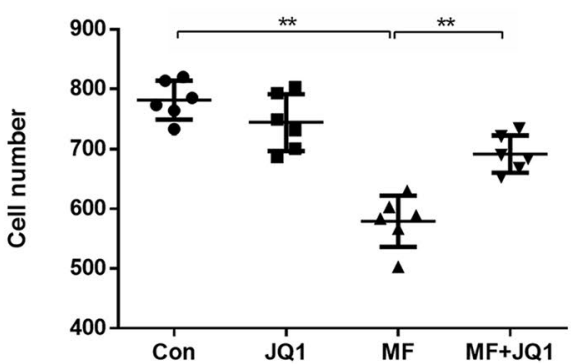

d

e
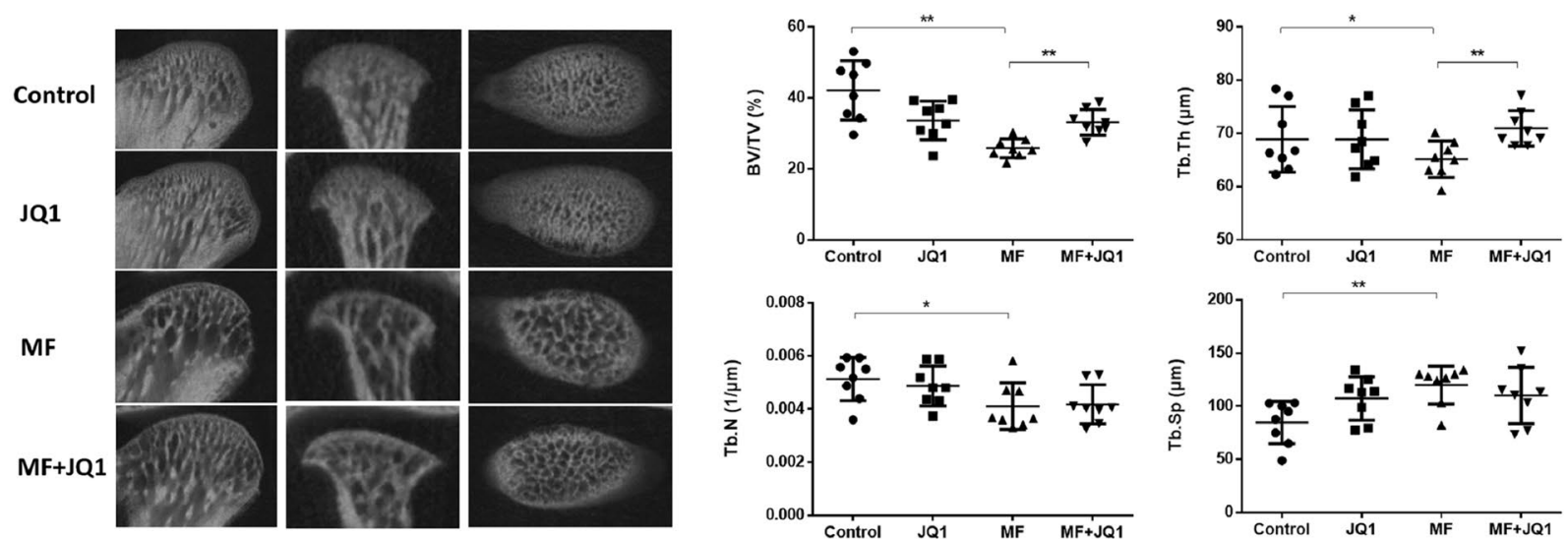

Fig. 1 JQ1 can relieve the condylar cartilage thinning and subchondral bone resorption induced by overloading mechanical stress. a HE-stained sections $(100 \times)$ of cartilage treated with $50 \mu \mathrm{M} J \mathrm{JQ} 1$ at 14 days $(n=6)$. Scale bar indicates $100 \mu \mathrm{m}$. Cartilage thickness is indicated (black bar). FP indicates proliferative zone, T indicates transition zone, and $\mathrm{H}$ indicates hypertrophic zone. $\mathbf{b}$ Quantification of cartilage thickness of the samples shown in $1 \mathrm{~A}(n=6)$. Error bars indicate the standard deviation. (\#\# $P<0.01$ vs MF group; ${ }^{* *} P<0.01$ vs control group). c Quantification of chondrocytes of the samples shown in $1 \mathrm{~A}(n=6)$. Error bars indicate the standard deviation $\left({ }^{*} P<0.05\right.$; $\left.{ }^{* *} P<0.01\right)$. $\mathbf{d}$ Representative images showing trabecular architecture by micro-computed tomography $(\mathrm{mCT}$ ) reconstruction in the subchondral bone. e $\mathrm{mCT}$ measurements for the indicated parameters in the subchondral bone. BV/TV, Tb.Th, Tb.N, and Tb.Sp were determined by mCT analysis $(n=8)$. Error bars indicate the standard deviation. ${ }^{*} P<0.05$; $\left.{ }^{* *} P<0.01\right)$. MF, mechanical force 


\section{(See figure on next page.)}

Fig. 2 BRD4 inhibition can alleviate the expression of inflammatory factors induced by excessive mechanical stress and IL-1 $\beta$. The concentration of JQ1 used in animal experiment is $50 \mu \mathrm{M}$. a qRT-PCR analysis of Tnf- $a, \|-1 \beta$, and $I I-6$ in condylar cartilage at 14 days $(n=6)$. b Immunohistochemical analysis of TNF- $\alpha$ and IL-1 $\beta$ in condylar cartilage $(200 x)$ at 14 days $(n=12)$. TNF- $a$ expressing and IL-1 $\beta$ expressing chondrocytes are denoted (arrow). c qRT-PCR analysis of $I-1 \beta, B r d 4, I I-6$, Tnf- $a$ in chondrocytes from samples treated as indicated. Error bars indicate the standard deviation $\left({ }^{*} P<0.05 ;{ }^{* *} P<0.01\right)$

\section{Excessive mechanical stress increases BRD4 occupancy} on inflammation-related genes promoter regions in vivo Previously we demonstrated that BRD4 inhibition showed significant protective effect on cartilage thinning, subchondral bone resorption, and inflammatory factors expression induced by mechanical stress. As the mechanism of BRD4 inhibition is blocking the interaction between BRD4 and acetylated histones sites on DNA, we next attempt to investigate the BRD4 genome-wide occupancy profile to fully uncover the changes of BRD4's binding to DNA under excessive mechanical stress. We performed chromatin immunoprecipitation sequencing (ChIP-seq) with BRD4 antibody using cartilage samples from control and MF groups.

ChIP-seq analysis for BRD4 revealed 40,722 peaks and 15,423 peaks in control and MF group, respectively (Fig. 3a). These peaks were distributed widely over the genic and intergenic regions. Approximately $45 \%$ of BRD4 peaks were found in genic regions, while the rest of BRD4 peaks $(\sim 55 \%)$ were distributed over the intergenic regions (Fig. 3a). There was increased BRD4 binding surrounding the transcription start site (TSS) at the genomewide level between Con and MF groups (Fig. 3b, c). The KEGG pathway and GO analysis provided us some clues of BRD4's regulation on inflammatory response. The top ten most significant enrichment pathways were obtained from the increased BRD4-binding peaks on the promoter region after overloading mechanical stress (Fig. 3d). Two inflammation-related pathways were included which were inflammatory mediator regulation of TRP channels (ranked 3rd) and MAPK signaling pathway (ranked 7 th). Genes such as $I l-1 \beta, M y c, N f-\kappa b$ were involved (Additional file 1: Table S2) which may transcribe under mechanical stress and cause joint damage aggravating. The top four most significant enrichment GO terms which are related to inflammation are regulation of stress-activated MAPK cascade, chronic inflammatory response, leukocyte aggregation, regulation of cytokine production involved in inflammatory response (Fig. 3e). Genes included are listed in Additional file 1: Table S3.

\section{Excessive mechanical stress increases BRD4 binding to the H3K27ac on the promoter region of Trem 1 in vivo}

As BRD4 can recognize the H3K27ac site to regulate gene transcription [31-33], we also performed ChIP-seq with
H3K27ac antibody using cartilage samples from control and mechanical force groups. The aim is to identify the overlapped increased BRD4-binding and H3K27ac-binding sites in the chondrocytes after overloading mechanical stress and to further investigate the role of BRD4 in mechanical stress-induced TMJ pathological changes. In total, 522 genes were identified to have increasing H3K27ac binding and 346 genes were identified to have increasing BRD4 binding in the promoter region after overloading mechanical stress (Fig. 4a). The 17 overlapped genes which have increased binding of BRD4 or H3K27ac were listed in the heat map (Fig. 4b). The details of the 17 genes are listed in Additional file 1: Table S4.

Through literature review, we found 4 genes are related to bone or joint diseases which are Cystatin A, Mir-181d, Mir-181c, and Trem1. Cystatin A (CSTA) and desmoplakin (DSP) interaction was found in human osteoarthritis, but the mechanism is unclear [34]. Mir-181d and Mir-181c are two miRNAs which are related to bone metabolism. Mir-181d can inhibit osteogenic differentiation of hBMSCs [35]. Mir-181c is the potential biomarkers for prognosis and diagnosis of osteoporosis [36]. Trem1, triggering receptor expressed on myeloid cells 1 , was found to play a role in the regulation of inflammation. It was strongly upregulated in inflamed compared with normal/reactive areas of osteoarthritis synovial membrane, and it plays a critical in OA development through regulation of NF- $\mathrm{kB}$ signaling [29, 37, 38]. Gene tracks showed that overloading mechanical stress can increase BRD4 and Н3К27ac co-occupancy on the promoter region of Trem1 (Fig. 4c). As we focused on the anti-inflammatory effect of BRD4 inhibition and Trem1 is a pro-inflammation factors with increased BRD4 binding after overloading, we hypothesized that BRD4-regulated inflammatory response may be mediated by Trem1.

\section{BRD4 inhibition reduces expression of inflammatory factors via regulating Trem 1}

It is found that both BRD4 inhibitor JQ1 and siBRD4 can decrease the expression of Trem1 mRNA induced by the mechanical force in vivo or IL-1 $\beta$ in vitro (Fig. 5ac). Next, we built the siTREM1 to investigate whether TREM1 can regulate other inflammatory factors (Additional file 1: Fig. S5). We found that in vitro IL-1 $\beta$ induced increase of endogenous $I l-1 \beta, I l-6$, and $\operatorname{Tn} f-\alpha$ mRNA was reduced by siTREM1 (Fig. $5 \mathrm{~d}$ ). Combined 
a

Relative mRNA level of TNF-a

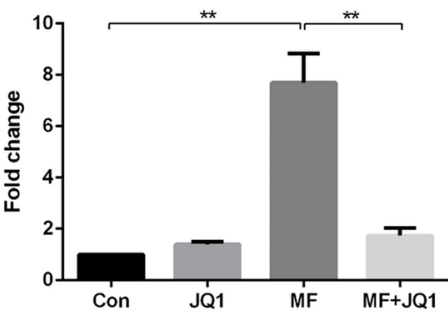

Relative mRNA level of IL-1 $\beta$



Relative mRNA level of IL-6

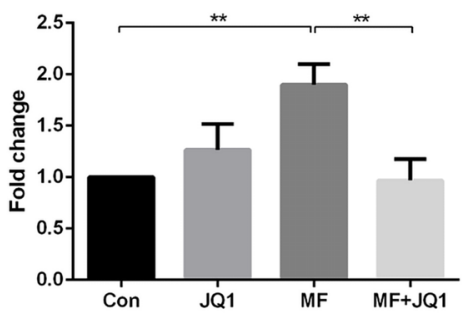

b

TNF-a

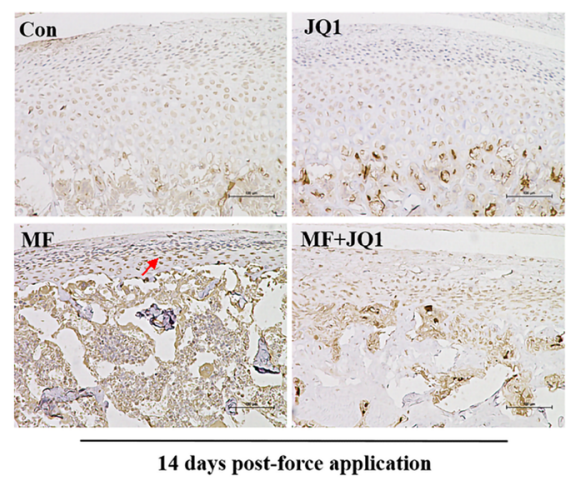

\begin{tabular}{|c|c|c|c|c|c|}
\hline Score & 0 & 1 & 2 & 3 & P value \\
\hline Con & 3 & 9 & 0 & 0 & - \\
\hline JQ1 & 4 & 7 & 1 & 0 & $>0.05$ vs Con \\
\hline MF & 0 & 2 & 8 & 2 & $<0.01$ vs Con \\
\hline MF+JQ1 & 2 & 9 & 1 & 0 & $<0.01$ vs MF $^{*}$ \\
\hline
\end{tabular}

IL-1ß



14 days post-force application

c

\begin{tabular}{|c|c|c|c|c|c|}
\hline Score & 0 & 1 & 2 & 3 & P value \\
\hline Con & 8 & 4 & 0 & 0 & - \\
\hline JQ1 & 7 & 5 & 0 & 0 & $>0.05$ vs Con \\
\hline MF & 0 & 1 & 3 & 8 & $<0.01$ vs Con \\
\hline MF+JQ1 & 8 & 4 & 0 & 0 & $<0.01$ vs MF $^{*}$ \\
\hline
\end{tabular}
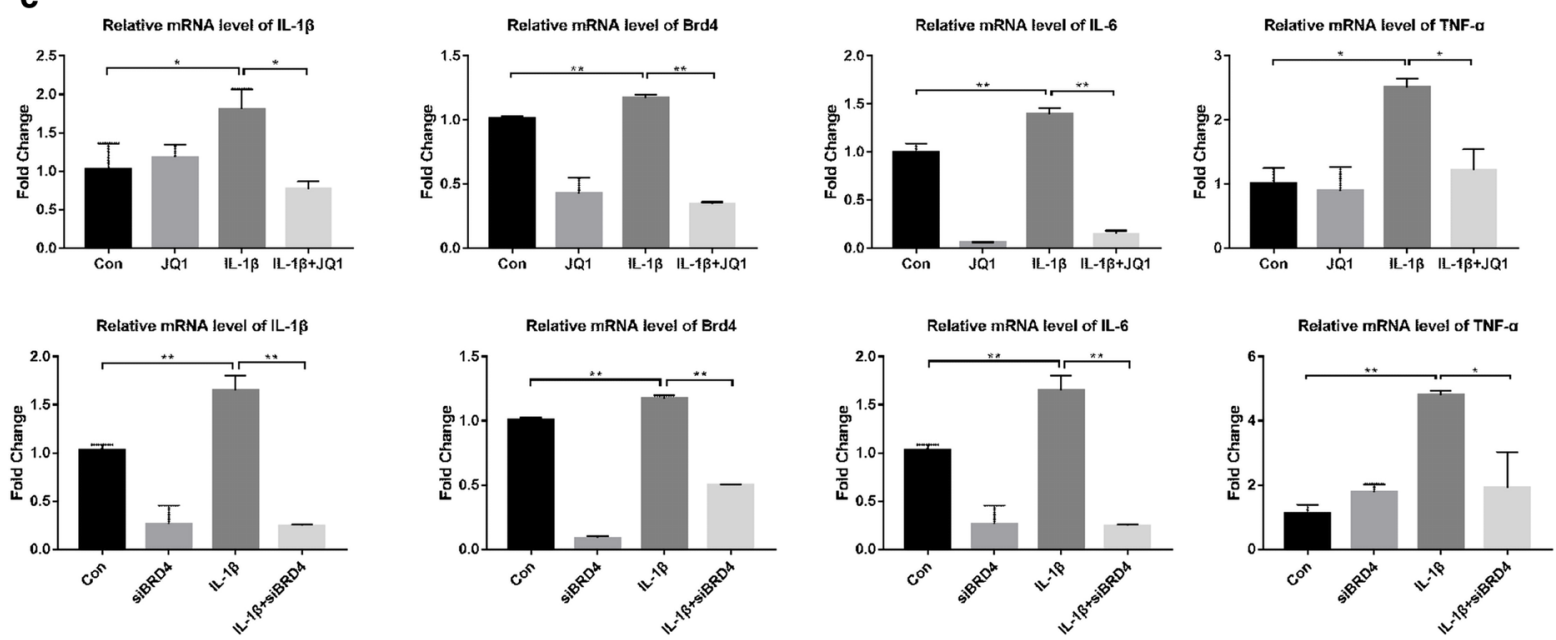


\section{(See figure on next page.)}

Fig. 3 Overloading mechanical stress increases BRD4 occupancy on inflammation and stress-related genes promoter region. a Genome-wide distribution of BRD4 in condylar cartilage from control (Con) and mechanical force (MF) group. The numbers on the top represent total peak numbers. $\mathbf{b}$ Heat maps of BRD4 occupancy on the promoter region (TSS $\pm 5 \mathrm{~kb}$ ), aligned by the degree of BRD4 signal intensity in condylar cartilage from control (Con) and mechanical force (MF) group. c ChIP-seq tag distribution of BRD4 surrounding the TSS ( $\pm 5 \mathrm{~kb}$ ) of the whole genome in the condyles from Con and MF groups. d KEGG pathway analysis of increased BRD4-binding peaks in rat TMJ cartilage after overloading mechanical stress. The top ten most significant enrichment pathways and the related details were shown. e GO analysis of increased BRD4-binding peaks in rat TMJ cartilage after overloading mechanical stress. The top six most significant enrichment GO terms which are related to inflammation and the details were shown

with previous findings (Fig. 2), we concluded that BRD4 inhibition can regulate TREM1-mediated inflammatory response under mechanical stress or IL-1 $\beta$ treatment.

\section{Discussion}

In our TMJ OA model induced by compressive stress, we found marked inflammatory cells infiltration in the subchondral bone near dilated blood vessels and increased inflammatory cytokines expression such as Tnf- $\alpha, I l-1 \beta$, and Il-6. Furthermore, we demonstrated that BRD4mediated inflammatory responses play an important role in TMJ OA for the first time. Recent experimental data have shown that subchondral bone may have a substantial role in the OA process, as a source of inflammatory mediators implicated in the OA pain process and in the degradation of the deep layer of cartilage [39]. Clinical studies also demonstrated a direct association between joint inflammation and the progression of OA $[2,39]$. Thereby, our results demonstrated that the inflammation is an important pathological change that cannot be ignored and targeting joint inflammation will help to slow the progression of TMJ OA. BRD4 inhibition such as JQ1 can be a potential therapeutic agent for TMJ OA.

In this study, to explore the mechanism under the protective effect of BRD4 inhibition on TMJ under mechanical stress, we used ChIP-seq to conduct highthroughput screening. With this technology, we can fully uncover the changes of BRD4's binding to DNA under excessive mechanical stress. The BRD4 peaks decreased after excessive mechanical stress loading which means the number of BRD4's binding sites decreased at the genome-wide level. But the KEGG pathway and GO analysis showed enriched BRD4 binding to several inflammation-related pathways and biological process which means BRD4 may binds more intensively to specific DNA region. Two inflammation-related pathways are screened out from BRD4 ChIP-seq analysis which are MAPK signaling pathway and inflammatory mediator regulation of TRP channels. The excessive mechanical stress can also increase BRD4 occupancy on the other inflammationrelated genes which are involved in chronic inflammatory response, leukocyte aggregation, and regulation of cytokine production involved in inflammatory response.
Our results showed that BRD4 could play a comprehensive role in regulating mechanical stress-induced TMJ inflammation.

Trem1 was screened out from BRD4 and H3K27ac ChIP-seq. TREM1 is an immunoglobulin-like cell surface receptor first found in polymorphonuclear cells [40]. Its activation leads to the NF- $\mathrm{KB}$ signaling pathway activation and the production of multiple proinflammatory cytokines and chemokines [41, 42]. Dan reported that TREM1 is a therapeutic target to inhibit the inflammatory response in rheumatoid arthritis [38]. Tang reported that knockdown of Trem 1 suppresses IL-1 $\beta$-induced chondrocyte injury [29]. Clinical study showed TREM1 was strongly upregulated in the inflamed synovial membrane [37] and cartilage [29] from TMJ OA patients. In this study, we found excessive mechanical stress can increase BRD4 and H3K27ac co-occupancy on the promoter region of Trem1 and increase TREM1 expression. We furthermore confirmed BRD4 can regulate TREM1 expression induced by both mechanical force or IL-1 $\beta$ and TREM1's ability to regulate inflammation. Our results first revealed the relation between BRD4 and TREM1 and proved that BRD4 inhibition alleviates TMJ inflammation under mechanical stress partly via regulating TREM1 (Fig. 6).

The limitation of this study is that we did not prove TREM1's role in mechanical stress-induced TMJ OA in vivo. Although we confirmed the BRD4 can regulate TREM1 and we performed in vitro study to prove TREM1 can regulate downstream inflammatory genes expression under IL-1 $\beta$ treatment, we are still not ensured whether TREM1 plays an important role in the pathological changes of TMJ under mechanical stress loading. If we can confirm that, the role of BRD4-TREM1 axis in TMJ OA can be more convinced. Whether other screened out genes and pathways anticipate in the protective effect of BRD4 inhibition on TMJ OA also needs further research.

\section{Conclusions}

In summary, this study for the first time focused on the effects of BRD4 inhibition on excessive mechanical stress-induced TMJ pathological changes and explored 
a BRD4 Genome-wide distribution

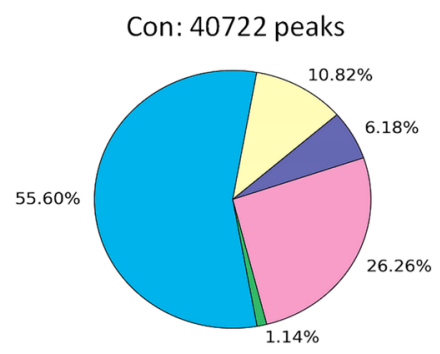

C


b

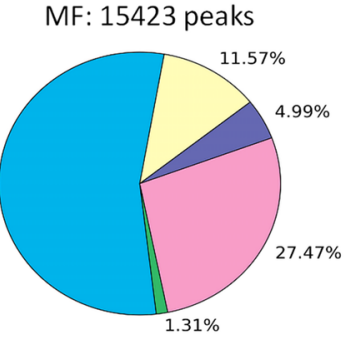

$1.31 \%$



e

Sig GO terms of DE gene-BP

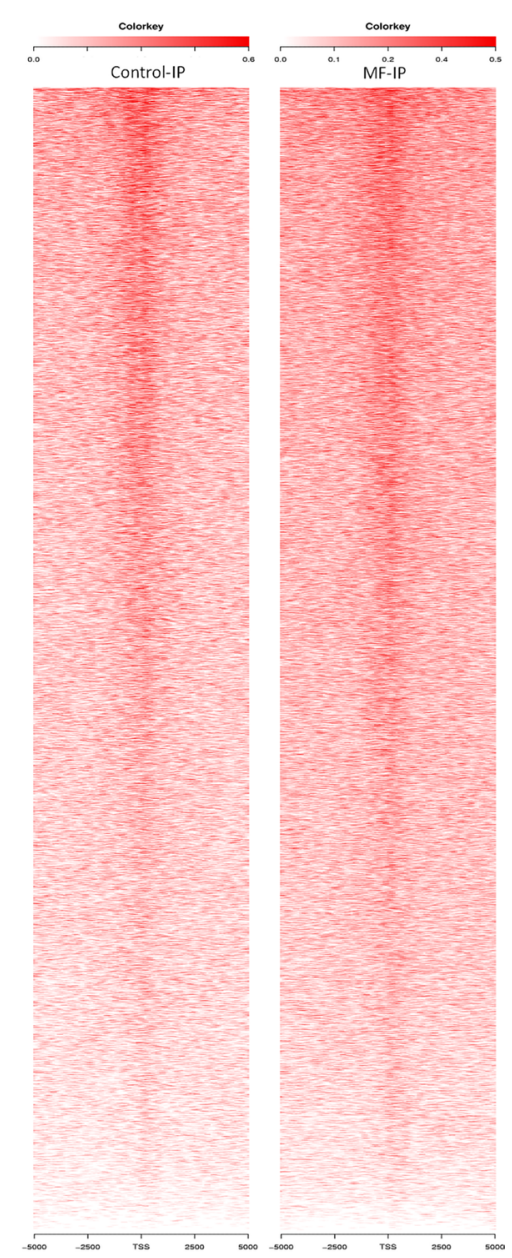

BRD4 occupancy on the promoter region

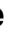

GO :0032827 Regulation of stress-activated MAPK cascade [9]

GO :0002544 Chronic inflammatory response [3]

GO :0070486 Leukocyte aggregation [2]

GO :1900015 Regulation of cytokine production involved in inflammatory response [3]

GO :0051403 Stress-activated MAPK cascade [9]

GO :0002534 Cytokine production involved in inflammatory response [3]
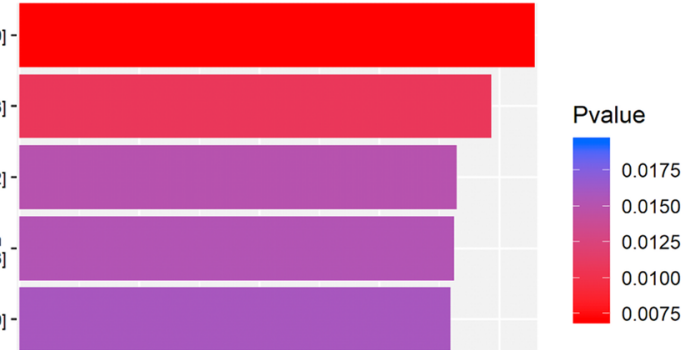

$$
-
$$

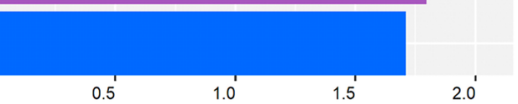

Enrichment Score (-log10(Pvalue)) 

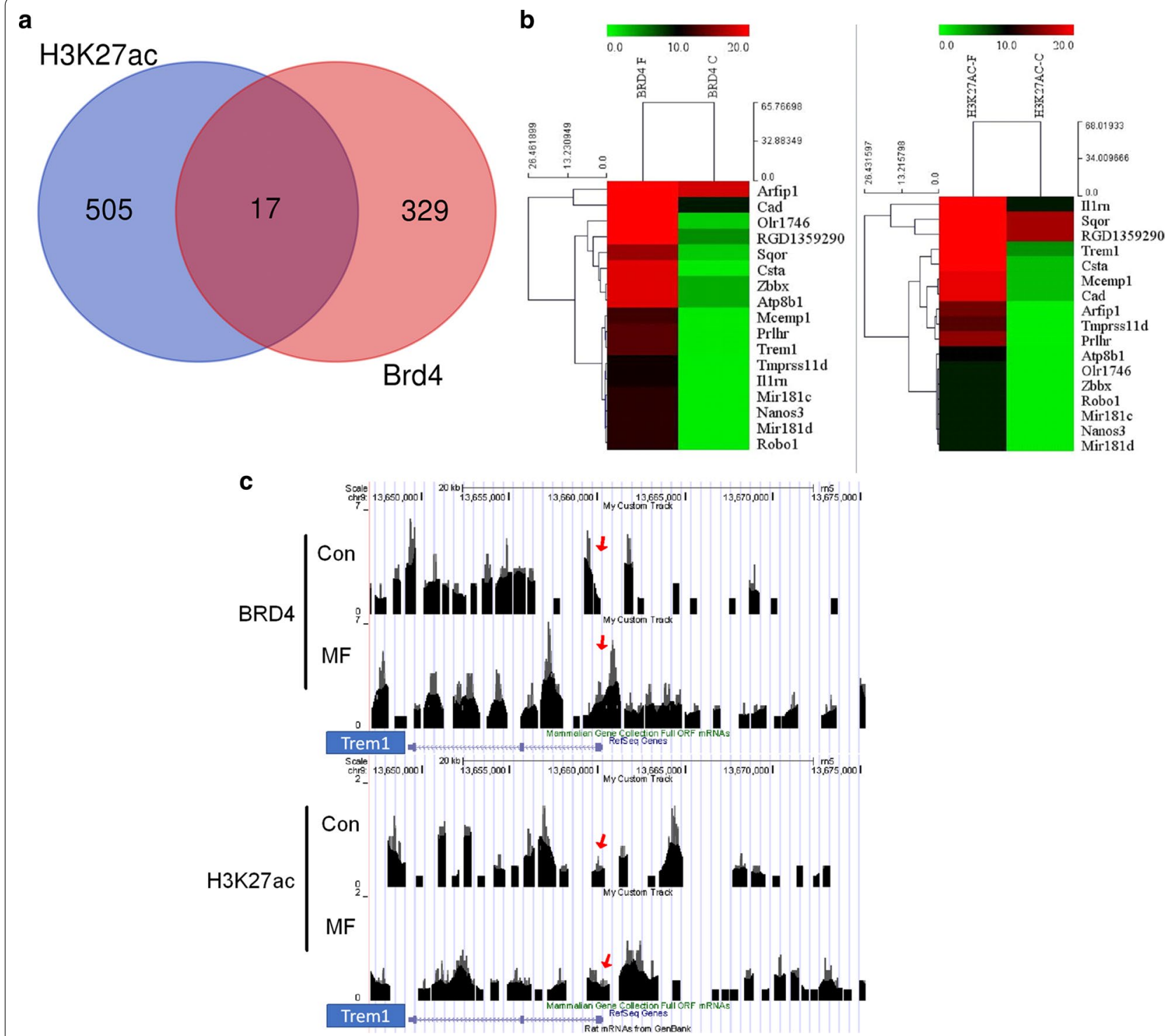

Fig. 4 Overloading mechanical stress increases BRD4 binding to the H3K27ac on the promoter region of 17 genes including Trem 1. a Venn diagram depicting the number of increased enriched genes binding to BRD4 or H3K27ac after overloading stress. b The 17 overlapped genes which have increased binding of BRD4 and H3K27ac were listed in the heat map. c Gene tracks of ChIP-seq signal for BRD4 and H3K27ac at the Trem 1 gene loci in control (top) and MF group (bottom). The y-axis shows the relative enrichment of the ChIP-seq signal. The $x$-axis depicts genomic position. Red arrow indicates the promoter region of Trem1

the mechanism. We concluded that BRD4 inhibition can protect TMJ from cartilage thinning and subchondral bone resorption and exerts an anti-inflammatory effect via regulating TREM1 under mechanical stress. Our findings highlight the protective effect of BRD4 inhibition on TMJ OA and suggest that JQ1 might be a novel therapeutic agent against TMJ OA.

\section{Materials and methods}

\section{Animal models}

Experimental protocols were reviewed and approved by the Animal Care and Use Committee of Nanjing University. This study also complied with Animal Research: Reporting In Vivo Experiments (ARRIVE) guidelines for preclinical animal studies. All animals were housed in 


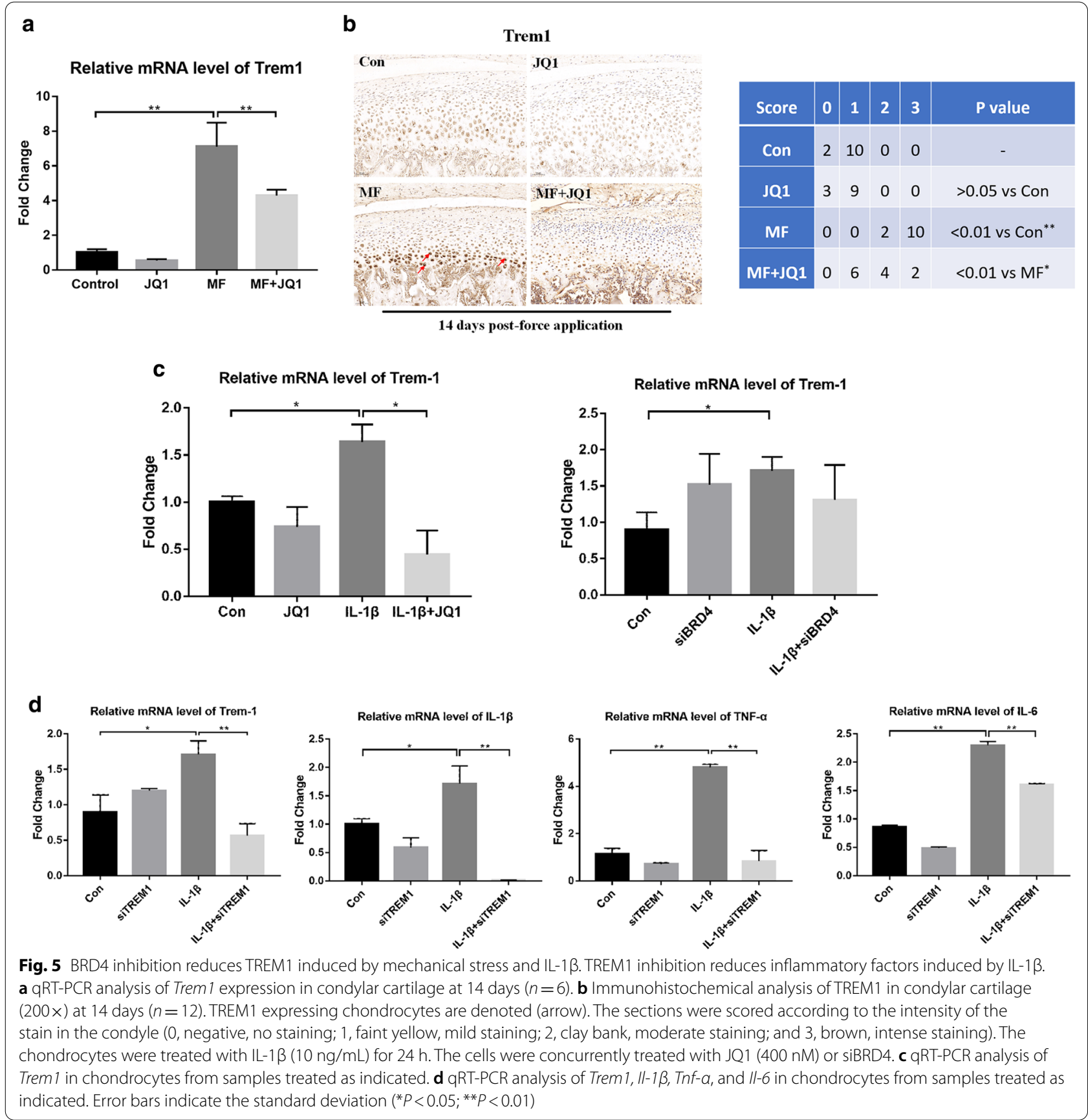

an approved facility at Nanjing University. One hundred and sixty-eight condyles from eighty-four 8-wk-old male Sprague-Dawley rats were used in this study. The animals were housed in a light- and temperature-controlled room and given unrestricted access to food and water during the experimental period. Before the start of the experiment, all of the animals were acclimated to their surroundings for 1 day. All of the animals were randomly divided into non-mechanical force (Control) group and mechanical force (MF) group. All of the rats in the MF groups were loaded with $80 \mathrm{~g}$ compressive mechanical force on the first day of the experiment as previously described [6, 43]. Briefly, 2 hooks were made with 0.025 inch stainless steel wire after the lower incisors of the rats were cleaned; then, resin was shaped into a sphere so that an undercut was formed and the hooks could not come out. The anchorage jigs were made of copper wires and were placed around the neck and arms. A rubber band 


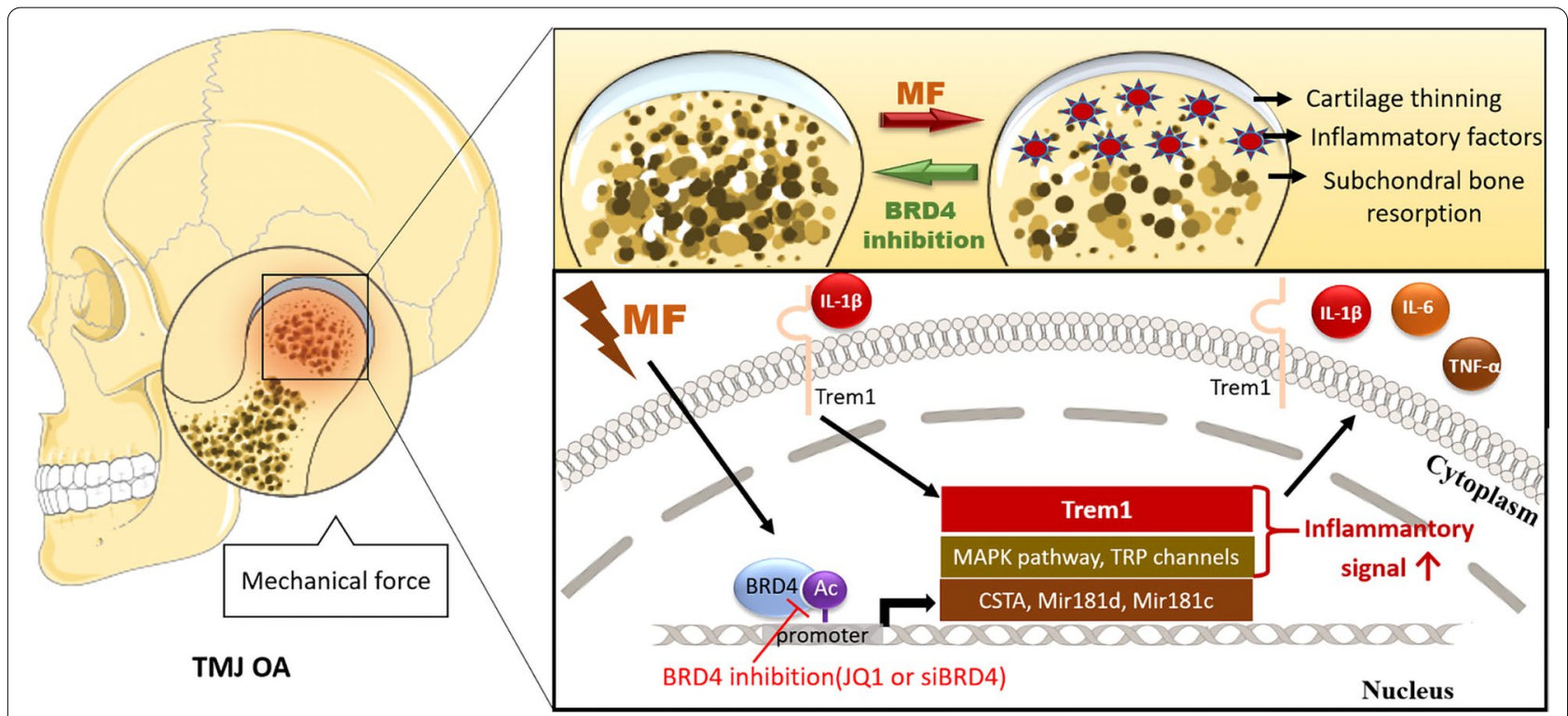

Fig. 6 The role of BRD4 in the mechanical stress-induced TMJ OA pathological changes and inflammatory response of chondrocytes. The excessive mechanical stress is an important etiological factor of TMJ OA. It can cause cartilage thinning, subchondral bone destruction, and joint inflammation. BRD4 inhibition can alleviate the TMJ pathological changes induced by mechanical stress. The underlying mechanism is shown in the schematic diagram of the chondrocyte. At the cellular level, the binding of BRD4 to the promoter region of the inflammation-related genes and microRNA increased under mechanical stress stimulation. We confirmed the binding enhanced the transcription of Treml and inflammatory factors such as $I-1 \beta$, Tnf- $a$, and $I I-6$. After these receptor and inflammatory factors are expressed, they aggravated the inflammatory response of the chondrocytes. IL-1 $\beta$-induced inflammation is TREM1-dependent, and both IL-1 $\beta$ and TREM1 are regulated by BRD4. Our study indicates that BRD4 plays an important role in mechanical stress-induced TMJ pathological changes and especially cartilage inflammatory response. TREM 1 is involved in the BRD4's epigenetic regulation of inflammation

was tied between the anchorage jig and the hook on the same side to load $80 \mathrm{~g}$ of compressive mechanical force upward and backward on each side (Additional file 1: Fig. S2). The rats in MF group wore the loading appliance for $14 \mathrm{~d}$. None of the rats displayed signs of disability, and all of the animals received the same standardized diet throughout the procedure. (+/-)-JQ1(SML0974, Sigma) $(50 \mu \mathrm{M})$ was injected locally into the temporomandibular joint (TMJ) on one side, and vehicle (dimethyl sulfoxide; Solarbio) was injected on the other side as described [44] at the 4th, 7th, and 11th day after mechanical force loading. A tailored micro-injector was inserted just below the zygomatic arch between the eye and ear until the outer surface of the mandibular ramus was reached. The orientation of the needle head was adjusted to enable the needle to pass along the bone wall and finally reach the TMJ region.

\section{Tissue preparation and histological staining}

All of the animals and their age-matched controls were killed at the 14th day by cervical dislocation under diethyl ether anesthesia. TMJ specimens were harvested. These specimens were fixed in formalin for $24 \mathrm{~h}$ and decalcified in ethylenediaminetetraacetic acid (EDTA) for 8 weeks at $4{ }^{\circ} \mathrm{C}$. Then, they were dehydrated, embedded in paraffin, and sectioned through the TMJ sagittal plane to make 5 - $\mu$ m-thick serial sections. The paraffin sections were deparaffinized in xylene and rehydrated through graded alcohols. Then, 3 slides of central sagittal sections from each sample were stained with hematoxylin and eosin (HE) for histological assessment. The thickness of the mandibular condylar cartilage and number of osteoclasts were measured by computerized morphometry, which was carried out by a single observer who was blinded to the experimental protocol. All images were captured by an Olympus XI 70 microscope equipped with an Olympus Magna Fire digital camera and were analyzed using a computerized digital image analysis system (Image-Pro Plus, version 6.0; MediaCybernetics). The condylar cartilage was equally divided into 3 parts: the anterior, middle, and posterior thirds, as previously described [6]. We mainly focused on changes in the middle third, which is the main load-bearing area based on the direction of force application (Additional file 1: Fig. S3). The thickness of the cartilage was measured in 3 squares located at the quartering points of the center third of the cartilage [45]. The mean of the values for the 3 squares in each section was used for further statistical analysis. 


\section{Immunohistochemistry}

Immunohistochemistry was performed as previously described [6]. In brief, 5 - $\mu$ m-thick sections (3 sections per specimen) were prepared according to standard protocols for HE staining. Rabbit anti-IL-1 $\beta$ (sc-7884, Santa Cruz, USA), Rabbit anti-TNF- $\alpha$ (ab6671, Abcam, Hong Kong), and Rabbit anti-TREM1 (AF8226, Beyotime, Shanghai) served as the primary antibodies, and goat anti-rabbit horseradish peroxidase-conjugated IgG served as the secondary antibody. The slides were incubated with primary antibodies overnight at $4{ }^{\circ} \mathrm{C}$ and with secondary antibodies for $30 \mathrm{~min}$ at $37^{\circ} \mathrm{C}$. Diaminobenzidine (SP immunochemistry kit; Boster Biological Technology, Ltd.) was then applied, followed by counterstaining with hematoxylin. Image acquisition was performed using the same method used for the HE-stained sections. The immunohistochemical measurements were taken by 2 observers. The sections were scored according to the intensity of the stain in the condyle $(0$, negative, no staining; 1, faint yellow, mild staining; 2, clay bank, moderate staining; and 3, brown, intense staining). Interobserver differences were reconciled through microscope conferencing.

\section{Micro-CT analysis}

Routine calibration was performed once per week using a three-point calibration phantom corresponding to a density range from air to cortical bone. The rats TMJs were scanned with an ex vivo micro-CT system (GE eXplore Locus SP, London) at $80 \mathrm{kV}$ and $80 \mathrm{~mA}$. The X-ray image was reconstructed with an isotropic voxel size of $9 \mu \mathrm{m}$. After acquiring the radiographic data, images were processed using the Health Care MicroView ABA software. The value of BV/TV, Tb.N, Tb.Th, and Tb.Sp was measured.

\section{Mandibular chondrocyte culture}

Ninety-six mandibular condylar cartilage was isolated from forty-eight 3-wk-old male Sprague-Dawley rats and subsequently minced. After the primary condylar chondrocytes were digested with $0.25 \%$ trypsin (Gibco/ Invitrogen) for $30 \mathrm{~min}$ and with $0.2 \%$ type II collagenase (Sigma-Aldrich) for $3 \mathrm{~h}$, they were rinsed 3 times and prepared as a single cell suspension. The primary condylar chondrocytes were counted with a hemocytometer. The chondrocytes were seeded at a density of $1 \times 10^{5}$ cells $/ \mathrm{cm}^{2}$ in a humidified atmosphere of $37{ }^{\circ} \mathrm{C}$ and $5 \%$ $\mathrm{CO}_{2}$. The cells were cultured to the third generation for subsequent experiments. The cultured cells were divided into 4 groups according to the treatment they received. These groups included the control, JQ1 (cells treated with $400 \mathrm{nM}$ ), IL-1 $\beta$ (cells treated with $10 \mathrm{ng} / \mathrm{mL}$ IL-1 $\beta$ ), IL-1 $\beta+$ JQ1 (cells treated concurrently with $10 \mathrm{ng} / \mathrm{mL}$ IL-1 $\beta$ and $400 \mathrm{nM} \mathrm{JQ1).}$

\section{Small Interference RNA (siRNA) infection}

Small interfering RNA (siRNA) targeting BRD4 (siBRD4), small interfering RNA (siRNA) targeting TREM1 (siTREM1), and non-silencing siRNA were transformed into RNA oligos (siRNA), respectively. The BRD4 silencing sequences were 5'- GAACCUCCCUGAUUACUA UTTAUAGUAAUCAGGGAGGUUCTT-3'. The TREM1 silencing sequences were 5'-GACAGACUCUGGAUU AUAUTTA UAUAAUCCAGAGUCUGUCTT- $3^{\prime}$. The non-silencing siRNA was sense- $5^{\prime}$-UUCU CCGAAC GUGUCACGUTT-3 \& antisense-5'-ACGUGACAC GUUCGGAGAATT-3'. Lipofectamine 2000 reagent (Invitrogen, Carlsbad, CA, USA) was inserted into the mixing solution for $20 \mathrm{~min}$, and chondrocytes were harvested after $48 \mathrm{~h}$.

\section{Isolation of total RNA and qRT-PCR}

As the condyle is very small, we used three condyles from three different rats as a single cartilage sample for RNA extraction in the in vivo model. In the in vitro model, different groups of cells from a 6-well plate were collected. Total RNA was extracted using TRIzol (Invitrogen Life Technologies, CA, USA). The primers used are listed in Additional file 1: Table S1. All the genes were analyzed using a 7500 Real-Time PCR machine (Applied Biosystems, CA, USA) using glyceraldehyde-3-phosphate dehydrogenase (GAPDH) as an internal control. The results were analyzed as the relative quantification compared to the control group. Each experiment was conducted three times, and the average values were calculated.

\section{ChIP sequencing}

ChIP sequencing was performed on condylar cartilage samples from control and mechanical force group using BRD4 antibody $(39,909$, ACTIVE MOTIF, America) and H3K27ac antibody (ab4729, Abcam, the USA) by KangChen (KangChen Bio-tech Inc, Shanghai, China). The detailed method and results are available from the corresponding author upon reasonable request. The purity and concentration of DNA samples were determined with Qubit ${ }^{\circledR}$ Fluorometer. In total, 10 ng of DNA samples was prepared for Illumina sequencing. Size selection of $200-1500$ bp enriched product using AMPure XP beads. The completed libraries were quantified by Agilent 2100 Bioanalyzer. The libraries were denatured to generate single-stranded DNA molecules and sequenced on the Illumina HiSeq 4000 following the HiSeq 3000/4000 SBS Kit (300 cycles) protocol. After the sequencing platform generated the sequencing images, 
the stages of image analysis and base calling were performed using Off-Line Basecaller software (OLB V1.8). Sequence quality was examined using the FastQC software. After passing Solexa CHASTITY quality filter, the clean reads were aligned to Rat reference genome RN5 using BOWTIE (V2.1.0). Aligned reads were used for peak calling of the ChIP regions using MACS V1.4.2. Statistically significant ChIP-enriched regions (peaks) were identified by comparison of IP vs input or comparison to a Poisson background model, using a p value threshold of $10^{-4}$. Then the peaks were annotated by the nearest gene using the newest UCSC RefSeq database. To generate heat maps for BRD4 occupancy on promoters, BRD4 signals were displayed on the promoter regions (encompassing the TSS $\pm 5 \mathrm{~kb}$ ), from condyles in Con and MF groups. Rows are ranked by BRD4 signal intensity. The density of BRD4 ChIP-seq signals was determined for $\mathrm{TSS} \pm 5 \mathrm{~kb}$ region shown as the read count per million mapped reads. GO analysis and pathway analysis were performed using $\mathrm{R}$ language package ( $\mathrm{R}$ 3.5.0). ChIPseq profile was visualized using UCSC Genome Browser (http://genome.ucsc.edu/cgi-bin/hgGateway).

\section{Statistical analysis}

All measurements were repeated three times. The data are expressed as means and standard deviations. All statistical analyses were conducted using the two-tailed, unpaired $t$ test (two conditions) or one-way ANOVA. All data were analyzed with SPSS 16.0 software, and $\mathrm{P}<0.05$ was used to define statistical significance.

\section{Supplementary information}

The online version contains supplementary material available at https://doi. org/10.1186/s13148-021-01008-6.

\footnotetext{
Additional file 1: Figure S1. Detailed description and diagram of the in vivo and in vitro experimental design. Figure S2. Establishment of the TMJ-OA rat model. Figure S3. Anterior, middle, and posterior thirds of the condylar cartilage. Figure S4. BRD4 expression in normal articular chondrocytes infected with siBRD4, compared with negative control. Figure S5. TREM1 expression in normal articular chondrocytes infected with siTREM1, compared with negative control. Figure S6. Condylar cartilage thinning induced by mechanical stress at $4 \mathrm{~d}, 7 \mathrm{~d}$ and $14 \mathrm{~d}$. Figure S7. BET inhibitor JQ1 $(5 \mu \mathrm{M}, 10 \mu \mathrm{M}, 50 \mu \mathrm{M})$ can relieve the condylar cartilage thinning and reduce the expression of inflammatory factors induced by overloading mechanical stress. Figure S8. The Survival rate of condylar chondrocytes of rats treated with JQ1 and IL-1 $\beta$ of different concentration. Table S1. Primers Used in Real-Time RT-PCR. Table S2. KEGG pathway analysis of increased BRD4-binding peaks in rat TMJ cartilage after overloading mechanical stress. Table S3. GO analysis of increased BRD4-binding peaks in rat TMJ cartilage after overloading mechanical stress. Table S4. The details of 17 genes with increased BRD4 and H3K27ac overlapped bindings in the promoter region after overloading mechanical force.
}

\section{Abbreviations}

TMJ OA:Temporomandibular joint osteoarthritis; siRNA: Small interference RNA; ECM: Extracellular matrix; ARRIVE: Animal Research: Reporting In Vivo
Experiments; MF: Mechanical force group; Control: Control group; EDTA: Ethylenediaminetetraacetic acid; HE: Hematoxylin and eosin; BV/TV: The ratio of bone volume to tissue volume; Tb.N: Trabecular number; Tb.Th: Trabecular thickness; Tb.Sp: Trabecular separation; FP: Fibrous proliferative layer; $\mathrm{T}$ : Transition layer; H: Hypertrophic layer; TSS: Transcription start site; CSTA: Cystatin A; DSP: Desmoplakin.

\section{Acknowledgements}

The authors thank Dr. Wei Chen for his support to this study. All authors gave final approval and agree to be accountable for all aspects of the work.

\section{Authors' contributions}

Ziwei Huang contributed to conception, data analysis, and interpretation, drafted, and critically revised the manuscript; Ren Yang contributed to the design, data acquisition, and analysis, drafted, and critically revised the manuscript; Lu Zhang, Mengjiao Zhu, and Caixia Zhang contributed to data acquisition and analysis, drafted the manuscript; J. Wen contributed to data acquisition and analysis, critically revised the manuscript; $\mathrm{H}$. Li contributed to conception, design, data analysis, and interpretation, drafted, and critically revised the manuscript. All authors read and approved the final manuscript.

\section{Funding}

This research was supported by the Jiangsu Provincial Medical Youth Talent, National Natural Science Foundation of China (Grant 81670960), and Natural Science Foundation of Jiangsu Province (Grant BK20171123).

\section{Availability of data and materials}

The datasets used and/or analyzed during the current study are available from the corresponding author on reasonable request (lihuang76@nju.edu.cn).

\section{Ethics approval and consent to participate}

Experimental protocols were reviewed and approved by the Animal Care and Use Committee of Nanjing University. This study also complied with Animal Research: Reporting In Vivo Experiments (ARRIVE) guidelines for preclinical animal studies. This article does not contain any studies with human participants performed by any of the authors.

\section{Consent for publication}

Not applicable.

\section{Competing interests}

The authors declare that they have no competing interests.

\section{Author details}

${ }^{1}$ Department of Orthodontics, Nanjing Stomatological Hospital, Medical School of Nanjing University, Nanjing, China. ${ }^{2}$ Department of Orthodontics, School and Hospital of Stomatology, Tongji University, Shanghai Engineering Research Center of Tooth Restoration and Regeneration, Shanghai, China. ${ }^{3}$ Department of Orthodontics, Nanjing Stomatological Hospital, Medical School of Nanjing University, 30 Central Road, Nanjing 210008, China.

Received: 7 October 2020 Accepted: 7 January 2021

Published online: 15 January 2021

\section{References}

1. da Silva CG, Pacheco-Pereira C, Porporatti AL, Savi MG, Peres MA, FloresMir $\mathrm{C}$, et al. Prevalence of clinical signs of intra-articular temporomandibular disorders in children and adolescents: a systematic review and meta-analysis. J Am Dent Assoc (1939). 2016;147(1):10-18.e8.

2. Tanaka E, Detamore MS, Mercuri LG. Degenerative disorders of the temporomandibular joint: etiology, diagnosis, and treatment. J Dent Res. 2008;87(4):296-307.

3. Ahmad M, Schiffman EL. Temporomandibular joint disorders and orofacial pain. Dent Clin N Am. 2016;60(1):105-24.

4. Kartha S, Zhou T, Granquist EJ, Winkelstein BA. Development of a rat model of mechanically induced tunable pain and associated temporomandibular joint responses. J Oral Maxillofac Surg Off J Am Assoc Oral Maxillofac Surg. 2016;74(1):54.e1-10. 
5. Li H, Yang HS, Wu TJ, Zhang XY, Jiang WH, Ma QL, et al. Proteomic analysis of early-response to mechanical stress in neonatal rat mandibular condylar chondrocytes. J Cell Physiol. 2010;223(3):610-22.

6. Li H, Zhang XY, Wu TJ, Cheng W, Liu X, Jiang TT, et al. Endoplasmic reticulum stress regulates rat mandibular cartilage thinning under compressive mechanical stress. J Biol Chem. 2013;288(25):18172-83.

7. Zhang C, Lin S, Li T, Jiang Y, Huang Z, Wen J, et al. Mechanical forcemediated pathological cartilage thinning is regulated by necroptosis and apoptosis. Osteoarthr Cartil. 2017;25(8):1324-34.

8. Huang Z, Zhou M, Wang Q, Zhu M, Chen S, Li H. Mechanical and hypoxia stress can cause chondrocytes apoptosis through over-activation of endoplasmic reticulum stress. Arch Oral Biol. 2017;84:125-32.

9. Wen J, Jiang Y, Zhang C, Chen S, Li H. The protective effects of salubrinal on the cartilage and subchondral bone of the temporomandibular joint under various compressive mechanical stimulations. PLOS ONE. 2016;11(5):e0155514.

10. Zhu M, Zhou S, Huang Z, Wen J, Li H. Ca2+-dependent endoplasmic reticulum stress regulates mechanical stress-mediated cartilage thinning. J Dent Res. 2016;95(8):889-96.

11. Sorenson A, Hresko K, Butcher S, Pierce S, Tramontina V, Leonardi R, et al. Expression of Interleukin-1 and temporomandibular disorder: contemporary review of the literature. Cranio J Craniomandib Pract. 2018;36(4):268-72.

12. Rahmati M, Mobasheri A, Mozafari M. Inflammatory mediators in osteoarthritis: a critical review of the state-of-the-art, current prospects, and future challenges. Bone. 2016;85:81-90.

13. Rogers EL, Reynard LN, Loughlin J. The role of inflammation-related genes in osteoarthritis. Osteoarthr Cartil. 2015;23(11):1933-8.

14. Meng S, Zhang L, Tang Y, Tu Q, Zheng L, Yu L, et al. BET inhibitor JQ1 blocks inflammation and bone destruction. J Dent Res. 2014;93(7):657-62.

15. Belkina AC, Nikolajczyk BS, Denis GV. BET protein function is required for inflammation: Brd2 genetic disruption and BET inhibitor JQ1 impair mouse macrophage inflammatory responses. J Immunol (Baltimore, Md: 1950). 2013;190(7):3670-8.

16. Dey A, Chitsaz F, Abbasi A, Misteli T, Ozato K. The double bromodomain protein Brd4 binds to acetylated chromatin during interphase and mitosis. Proc Natl Acad Sci USA. 2003;100(15):8758-63.

17. Dey A, Yang W, Gegonne A, Nishiyama A, Pan R, Yagi R, et al. BRD4 directs hematopoietic stem cell development and modulates macrophage inflammatory responses. EMBO J. 2019;38(7):e100293.

18. Jiang Y, Zhu L, Zhang T, Lu H, Wang C, Xue B, et al. BRD4 has dual effects on the HMGB1 and NF-kappaB signalling pathways and is a potential therapeutic target for osteoarthritis. Biochim Biophys Acta Mol Basis Dis. 2017;1863(12):3001-15.

19. Xiao Y, Liang L, Huang M, Qiu Q, Zeng S, Shi M, et al. Bromodomain and extra-terminal domain bromodomain inhibition prevents synovial inflammation via blocking lkappaB kinase-dependent NF-kappaB activation in rheumatoid fibroblast-like synoviocytes. Rheumatology (Oxford, England). 2015;55:173-84

20. Perry MM, Durham AL, Austin PJ, Adcock IM, Chung KF. BET bromodomains regulate transforming growth factor-beta-induced proliferation and cytokine release in asthmatic airway smooth muscle. J Biol Chem. 2015;290(14):9111-21.

21. Kokkola T, Suuronen T, Pesonen M, Filippakopoulos P, Salminen A, Jarho EM, et al. BET inhibition upregulates SIRT1 and alleviates inflammatory responses. ChemBioChem Eur J Chem Biol. 2015;16(14):1997-2001.

22. Khan YM, Kirkham P, Barnes PJ, Adcock IM. Brd4 is essential for IL-1 betainduced inflammation in human airway epithelial cells. PLOS ONE. 2014;9(4):e95051.

23. Baud'huin M, Lamoureux F, Jacques C, Rodriguez Calleja L, Quillard T, Charrier C, et al. Inhibition of BET proteins and epigenetic signaling as a potential treatment for osteoporosis. Bone. 2017:94:10-21.

24. Park-Min KH, Lim E, Lee MJ, Park SH, Giannopoulou E, Yarilina A, et al. Inhibition of osteoclastogenesis and inflammatory bone resorption by targeting BET proteins and epigenetic regulation. Nat Commun. 2014;5:5418.

25. Gao LN, Feng QS, Zhang XF, Wang QS, Cui YL. Tetrandrine suppresses articular inflammatory response by inhibiting pro-inflammatory factors via NF-kappaB inactivation. J Orthop Res Off Publ Orthop Res Soc. 2016;34:1557-68

26. Duan Q, Mao X, Xiao Y, Liu Z, Wang Y, Zhou H, et al. Super enhancers at the miR-146a and miR-155 genes contribute to self-regulation of inflammation. Biochim Biophys Acta. 2016;1859(4):564-71.

27. Filippakopoulos P, Qi J, Picaud S, Shen Y, Smith WB, Fedorov O, et al. Selective inhibition of BET bromodomains. Nature. 2010;468(7327):1067-73.

28. Goldring MB, Otero M, Plumb DA, Dragomir C, Favero M, El Hachem K, et al. Roles of inflammatory and anabolic cytokines in cartilage metabolism: signals and multiple effectors converge upon MMP-13 regulation in osteoarthritis. Eur Cells Mater. 2011;21:202-20.

29. Tang J, Dong Q. Knockdown of TREM-1 suppresses IL-1 beta-induced chondrocyte injury via inhibiting the NF-kappaB pathway. Biochem Biophys Res Commun. 2017;482(4):1240-5.

30. Kapoor M, Martel-Pelletier J, Lajeunesse D, Pelletier JP, Fahmi H. Role of proinflammatory cytokines in the pathophysiology of osteoarthritis. Nat Rev Rheumatol. 2011;7(1):33-42.

31. Xiong $L, W u F, W u Q, X u L$, Cheung OK, Kang W, et al. Aberrant enhancer hypomethylation contributes to hepatic carcinogenesis through global transcriptional reprogramming. Nat Commun. 2019;10(1):335.

32. Huang $X$, Yan J, Zhang M, Wang $Y$, Chen $Y$, Fu X, et al. Targeting epigenetic crosstalk as a therapeutic strategy for EZH2-aberrant solid tumors. Cell. 2018;175(1):186-99.e19.

33. Lee JE, Park YK, Park S, Jang Y, Waring N, Dey A, et al. Brd 4 binds to active enhancers to control cell identity gene induction in adipogenesis and myogenesis. Nat Commun. 2017;8(1):2217.

34. Wang M, Liu C, Zhang Y, Hao Y, Zhang X, Zhang YM. Protein interaction and microRNA network analysis in osteoarthritis meniscal cells. Genet Mol Res GMR. 2013;12(1):738-46.

35. Xie Y, Hu JZ, Shi ZY. MiR-181d promotes steroid-induced osteonecrosis of the femoral head by targeting SMAD3 to inhibit osteogenic differentiation of hBMSCs. Eur Rev Med Pharmacol Sci. 2018;22(13):4053-62.

36. Ma J, Lin X, Chen C, Li S, Zhang S, Chen Z, et al. Circulating miR-181 C-5p and miR-497-5p are potential biomarkers for prognosis and diagnosis of osteoporosis. J Clin Endocrinol Metab. 2020;105(5):1445-60.

37. Lambert C, Dubuc JE, Montell E, Verges J, Munaut C, Noel A, et al. Gene expression pattern of cells from inflamed and normal areas of osteoarthritis synovial membrane. Arthr Rheumatol (Hoboken, NJ). 2014;66(4):960-8.

38. Fan D, He X, Bian Y, Guo Q, Zheng K, Zhao Y, et al. Triptolide modulates TREM-1 signal pathway to inhibit the inflammatory response in rheumatoid arthritis. Int J Mol Sci. 2016;17(4):498.

39. Berenbaum F. Osteoarthritis as an inflammatory disease (osteoarthritis is not osteoarthrosis!). Osteoarthr Cartil. 2013;21(1):16-21.

40. Bouchon A, Dietrich J, Colonna M. Cutting edge: inflammatory responses can be triggered by TREM-1, a novel receptor expressed on neutrophils and monocytes. J Immunol (Baltimore, Md: 1950). 2000;164(10):4991-5.

41. Tessarz AS, Cerwenka A. The TREM-1/DAP12 pathway. Immunol Lett. 2008;116(2):111-6.

42. Sharif $\mathrm{O}$, Knapp S. From expression to signaling: roles of TREM-1 and TREM-2 in innate immunity and bacterial infection. Immunobiology. 2008;213(9-10):701-13.

43. Teramoto M, Kaneko S, Shibata S, Yanagishita M, Soma K. Effect of compressive forces on extracellular matrix in rat mandibular condylar cartilage. J Bone Miner Metab. 2003;21(5):276-86.

44. Suzuki S, Itoh K, Ohyama K. Local administration of IGF-I stimulates the growth of mandibular condyle in mature rats. J Orthod. 2004;31(2):138-43.

45. Jiao K, Wang MQ, Niu LN, Dai J, Yu SB, Liu XD, et al. Death and proliferation of chondrocytes in the degraded mandibular condylar cartilage of rats induced by experimentally created disordered occlusion. Apoptosis Int J Program Cell Death. 2009;14(1):22-30.

\section{Publisher's Note}

Springer Nature remains neutral with regard to jurisdictional claims in published maps and institutional affiliations. 